\title{
An Iterative Scheme with a Countable Family of Nonexpansive Mappings for Variational Inequality Problems in Hilbert Spaces
}

\author{
Yeol Je Cho' ${ }^{1}$ and Shenghua Wang, ${ }^{2,3}$ \\ ${ }^{1}$ Department of Mathematics Education and the RINS, Gyeongsang National University, \\ Chinju 660-701, South Korea \\ 2 School of Applied Mathematics and Physics, North China Electric Power University, \\ Baoding 071003, China \\ ${ }^{3}$ Department of Mathematics, Gyeongsang National University, Chinju 660-701, South Korea \\ Correspondence should be addressed to Shenghua Wang, sheng-huawang@hotmail.com \\ Received 13 December 2009; Accepted 8 February 2010 \\ Academic Editor: Jong Kim
}

Copyright (C) 2010 Y. J. Cho and S. Wang. This is an open access article distributed under the Creative Commons Attribution License, which permits unrestricted use, distribution, and reproduction in any medium, provided the original work is properly cited.

We introduce a new iterative scheme with a countable family of nonexpansive mappings for the variational inequality problems in Hilbert spaces and prove some strong convergence theorems for the proposed schemes.

\section{Introduction}

Let $H$ be a Hilbert space and $C$ be a nonempty closed convex subset of $H$. Let $F: H \rightarrow H$ be a nonlinear mapping. The classical variational inequality problem (for short, $\operatorname{VI}(F, C)$ ) is to find a point $x \in C$ such that

$$
\left\langle F\left(x^{*}\right), x-x^{*}\right\rangle \geq 0, \quad \forall x^{*} \in C .
$$

This variational inequality was initially studied by Kinderlehrer and Stampacchia [1]. Since then, many authors have introduced and studied many kinds of the variational inequality problems (inclusions) and applied them to many fields.

It is well known that, if $F$ is a strongly monotone and Lipschitzian mapping on $C$, then the $\operatorname{VI}(F, C)$ has a unique solution (see [2]). 
Let $T: H \rightarrow H$ be a mapping. Recall that a mapping $T: H \rightarrow H$ is nonexpansive if

$$
\|T x-T y\| \leq\|x-y\|, \quad \forall x, y \in H .
$$

The set of fixed points of $T$ is denoted by $F(T)$. Recently, the iterative methods for nonexpansive mappings and some kinds of nonlinear mappings have been applied to solve the convex minimization problems (see [3-7]).

A typical problem is to minimize a quadratic function over the set of the fixed points of a nonexpansive mapping on $H$ :

$$
\min _{x \in C} \frac{1}{2}\langle A x, x\rangle-\langle x, b\rangle,
$$

where $C$ is the fixed point set of a nonexpansive mapping $T$ on $\mathrm{H}, b$ is a given point in $H$ and $A$ is a strongly positive operator, that is, there is a constant $\bar{\gamma}>0$ such that

$$
\langle A x, x\rangle \geq \bar{\gamma}\|x\|^{2}, \quad \forall x \in H .
$$

Recently, for solving the variational inequality on $A$, Marino and $\mathrm{Xu}$ [8] introduced the following general iterative scheme:

$$
x_{n+1}=\left(I-\alpha_{n} A\right) T x_{n}+\alpha_{n} \gamma f\left(x_{n}\right), \quad \forall n \geq 0,
$$

where $A$ is a strongly positive linear bounded operator on $H, f$ is a contraction on $H$ and $\left\{\alpha_{n}\right\} \subset(0,1)$.

More precisely, they gave the following result.

Theorem MX (see [8, Theorem 3.4]). Let $\left\{x_{n}\right\}$ be generated by algorithm (1.5) with the sequence $\left\{\alpha_{n}\right\}$ satisfying the following conditions:

(C1) $\lim _{n \rightarrow \infty} \alpha_{n}=0$,

(C2) $\sum_{n=0}^{\infty} \alpha_{n}=\infty$,

(C3) either $\sum_{n=0}^{\infty}\left|\alpha_{n+1}-\alpha_{n}\right|<\infty$ or $\lim _{n \rightarrow \infty} \alpha_{n+1} / \alpha_{n}=1$.

Then the scheme $\left\{x_{n}\right\}$ defined by (1.5) converges strongly to an element $x^{*} \in C=F(T)$ which is the unique solution of the variational inequality (for short, $\operatorname{VI}(A-\gamma f, C))$ :

$$
\left\langle(A-\gamma f) x^{*}, x-x^{*}\right\rangle \geq 0, \quad \forall x \in C .
$$

Let $f: H \rightarrow H$ be a contraction with coefficient $0<\alpha<1$ and let $A, B: H \rightarrow H$ be two strongly positive linear bounded operators with coefficients $\bar{\gamma} \in(0,1)$ and $\beta>0$, respectively.

Motivated and inspired by the iterative sheme (1.5), Ceng et al. [9] introduced the following so-called hybrid viscosity-like approximation algorithms with variable parameters for nonexpansive mappings in Hilbert spaces. 
Theorem CGY1 (see [9, Theorem 3.1]). Let $0<\gamma \alpha<\beta$ and $\bar{\gamma} \in(0,1)$. Let $\left\{\lambda_{n}\right\}$ be a sequence in $(0,1)$ and $\left\{\mu_{n}\right\}$ be a sequence in $\left(0, \min \left\{1,\|B\|^{-1}\right\}\right)$. Starting with an arbitrary initial guess $x_{0} \in H$, generate a sequence $\left\{x_{n}\right\}$ by the following iterative scheme:

$$
x_{n+1}=\left(I-\lambda_{n+1} A\right) T x_{n}+\lambda_{n+1}\left[T x_{n}-\mu_{n+1}\left(B T x_{n}-\gamma f\left(x_{n}\right)\right)\right], \quad \forall n \geq 0 .
$$

Assume that

(i) $\lim _{n \rightarrow \infty} \lambda_{n}=0$,

(ii) $\sum_{n=1}^{\infty} \lambda_{n}=\infty$,

(iii) either $\sum_{n=1}^{\infty}\left|\lambda_{n+1}-\lambda_{n}\right|<\infty$ or $\lim _{n \rightarrow \infty} \lambda_{n} / \lambda_{n+1}=1$,

(iv) $(1-\bar{\gamma}) /(\beta-\gamma \alpha)<\lim _{n \rightarrow \infty} \mu_{n}=\mu<(2-\bar{\gamma}) /(\beta-\gamma \alpha)$.

Then the scheme $\left\{x_{n}\right\}$ defined by (1.7) converges strongly to an element $x^{*} \in C=F(T)$ which is the unique solution of the variational inequality (for short, $\operatorname{VI}(A-I+\mu(B-\gamma f), C))$ :

$$
\left\langle[A-I+\mu(B-\gamma f)] x^{*}, x-x^{*}\right\rangle \geq 0, \quad \forall x \in C .
$$

Theorem CGY2 (see [9, Theorem 3.2]). Let $0<\gamma \alpha<\beta$ and $\bar{\gamma} \in(0,1)$. Let $\left\{\lambda_{n}\right\}$ be a sequence in $(0,1)$ and $\left\{\mu_{n}\right\}$ be a sequence in $\left(0, \min \left\{1,\|B\|^{-1}\right\}\right)$. Starting with an arbitrary initial guess $x_{0} \in H$, generate a sequence $\left\{x_{n}\right\}$ by the following iterative scheme:

$$
x_{n+1}=\left(I-\lambda_{n+1} A\right) T_{[n+1]} x_{n}+\lambda_{n+1}\left[T_{[n+1]} x_{n}-\mu_{n+1}\left(B T_{[n+1]} x_{n}-\gamma f\left(x_{n}\right)\right)\right], \quad \forall n \geq 0 .
$$

Assume that

(i) $\lim _{n \rightarrow \infty} \lambda_{n}=0$,

(ii) $\sum_{n=1}^{\infty} \lambda_{n}=\infty$,

(iii) either $\sum_{n=1}^{\infty}\left|\lambda_{n+N}-\lambda_{n}\right|<\infty$ or $\lim _{n \rightarrow \infty} \lambda_{n} / \lambda_{n+N}=1$,

(iv) $(1-\bar{\gamma}) /(\beta-\gamma \alpha)<\lim _{n \rightarrow \infty} \mu_{n}=\mu<(2-\bar{\gamma}) /(\beta-\gamma \alpha)$.

In addition, assume that

$$
\begin{aligned}
C & =\bigcap_{i=1}^{N} F\left(T_{i}\right)=F\left(T_{1} T_{2} \cdots T_{N}\right)=F\left(T_{N} T_{1} \cdots T_{2}\right) \\
& =\cdots=F\left(T_{2} T_{3} \cdots T_{N} T_{1}\right) .
\end{aligned}
$$

Then the scheme $\left\{x_{n}\right\}$ defined by (1.9) converges strongly to an element $x^{*} \in C=F(T)$ which is the unique solution of the variational inequality (for short, $\operatorname{VI}(A-I+\mu(B-\gamma f), C))$ :

$$
\left\langle[A-I+\mu(B-\gamma f)] x^{*}, x-x^{*}\right\rangle \geq 0, \quad \forall x \in C .
$$

In this paper, motivated and inspired by the above research results, we introduce a new iterative process with a countable family of nonexpansive mappings for the variational inequality problem in Hilbert spaces. 
More precisely, let $H$ be a Hilbert space and $\left\{T_{i}\right\}_{i=1}^{\infty}$ be a countable family of nonexpansive mappings from $H$ to $H$ such that $C=\bigcap_{i=1}^{\infty} F\left(T_{i}\right) \neq \emptyset$. Let $f: H \rightarrow H$ be a contraction with coefficient $0<\alpha<1$ and $A, B$ be strongly positive linear bounded operators with coefficients $\eta \in(0,1)$ and $\beta>0$, respectively. Let $\left\{\lambda_{n}\right\}_{n=1}^{\infty} \subset(0,1)$ and $\left\{\alpha_{n}\right\}_{n=0}^{\infty} \subset(0,1]$ with $\alpha_{0}=1$. Take three fixed numbers $\gamma, \mu_{1}$ and $\mu_{2}$ such that $0<\gamma \alpha<\beta, \mu_{1} \in(0,1]$ and $\mu_{2} \in\left(\left(1-\eta \mu_{1}\right) /(\beta-\gamma \alpha), \min \left\{1,\|B\|^{-1},\left(2-\eta \mu_{1}\right) /(\beta-\gamma \alpha)\right\}\right]$. For any $x_{1} \in H$, generate the iterative scheme $\left\{x_{n}\right\}$ by

$$
\begin{aligned}
x_{n+1}= & \alpha_{n}\left[\left(I-\lambda_{n} \mu_{1} A\right) x_{n}+\lambda_{n}\left[x_{n}-\mu_{2}\left(B x_{n}-\gamma f\left(x_{n}\right)\right)\right]\right] \\
& +\sum_{i=1}^{n}\left(\alpha_{i-1}-\alpha_{i}\right) T_{i} x_{n}, \quad \forall n \geq 1 .
\end{aligned}
$$

We prove that the iterative scheme $\left\{x_{n}\right\}$ defined by (1.12) strongly converges to an element $x^{*} \in C$ which is the unique solution of the variational inequality (for short, $\operatorname{VI}\left(\mu_{1} A-\right.$ $\left.\left.I+\mu_{2}(B-\gamma f), C\right)\right)$ :

$$
\left\langle\left[\mu_{1} A-I+\mu_{2}(B-\gamma f)\right] x^{*}, x-x^{*}\right\rangle \geq 0, \quad \forall x \in C .
$$

\section{Preliminaries}

Let $H$ be a Hilbert space and $T$ be a nonexpansive mapping of $H$ into itself such that $F(T) \neq \emptyset$. For all $\widehat{x} \in F(T)$ and $x \in H$, we have

$$
\begin{aligned}
\|x-\widehat{x}\|^{2} & \geq\|T x-T \widehat{x}\|^{2}=\|T x-\widehat{x}\|^{2}=\|T x-x+(x-\widehat{x})\|^{2} \\
& =\|T x-x\|^{2}+\|x-\widehat{x}\|^{2}+2\langle T x-x, x-\widehat{x}\rangle
\end{aligned}
$$

and hence

$$
\|T x-x\|^{2} \leq 2\langle x-T x, x-\widehat{x}\rangle, \quad \forall \widehat{x} \in F(T), x \in H .
$$

Let $\left\{x_{n}\right\}$ be a sequence in a Hilbert space $H$ and let $x \in H$. Throughout this paper, $x_{n} \rightarrow x$ and $x_{n} \rightarrow x$ denote that $\left\{x_{n}\right\}$ strongly converges to $x \in H$ and $\left\{x_{n}\right\}$ converges weakly to a point $x \in H$, respectively.

Lemma 2.1 (see [10]). Let $C$ be a closed convex subset of a Hilbert space $H$ and $T$ be a nonexpansive mapping from $C$ into itself. Then $I-T$ is demiclosed at zero, that is,

$$
x_{n} \rightarrow x, \quad x_{n}-T x_{n} \longrightarrow 0 \quad \text { implies } x=T x
$$

The following lemma is an immediate consequence of the equality:

$$
\|x+y\|^{2}=\|x\|^{2}+2\langle y, x+y\rangle-\|y\|^{2}, \quad \forall x, y \in H
$$


Journal of Inequalities and Applications

Lemma 2.2. Let $H$ be a real Hilbert space. Then the following identity holds:

$$
\|x+y\|^{2} \leq\|x\|^{2}+2\langle y, x+y\rangle, \quad \forall x, y \in H
$$

Lemma 2.3 (see $[4,11]$ ). Let $\left\{s_{n}\right\},\left\{c_{n}\right\}$ be the sequences of nonnegative real numbers and let $\left\{a_{n}\right\} \subset$ $(0,1)$. Suppose that $\left\{b_{n}\right\}$ is a sequence of real numbers such that

$$
s_{n+1} \leq\left(1-a_{n}\right) s_{n}+b_{n}+c_{n}, \quad \forall n \geq 0 .
$$

Assume that $\sum_{n=0}^{\infty} c_{n}<\infty$. Then the following results hold.

(1) If $b_{n} \leq \beta a_{n}$, where $(\beta \geq 0)$, then $\left\{s_{n}\right\}$ is a bounded sequence.

(2) If one has

$$
\sum_{n=0}^{\infty} a_{n}=\infty, \quad \limsup _{n \rightarrow \infty} \frac{b_{n}}{a_{n}} \leq 0,
$$

then $\lim _{n \rightarrow \infty} S_{n}=0$.

Lemma 2.4 (see [8]). Let $H$ be a real Hilbert space, $f: H \rightarrow H$ be a contraction with coefficient $0<\alpha<1$ and $B$ be a strongly positive linear bounded operator with coefficient $\beta>0$. Then, for any $\gamma$ with $0<\gamma<\beta / \alpha$,

$$
\langle x-y,(B-\gamma f) x-(B-\gamma f) y\rangle \geq(\beta-\gamma \alpha)\|x-y\|^{2}, \quad \forall x, y \in H,
$$

that is, $B-\gamma f$ is strongly monotone with coefficient $\beta-\gamma \alpha$.

Lemma 2.5. Assume $A$ is a strongly monotone linear bounded operator on a Hilbert space $H$ with coefficient $\alpha>0$. Take a fixed number $\rho$ such that $0<\rho \leq\|A\|^{-1}$. Then $\|I-\rho A\| \leq 1-\rho \alpha$.

Proof. The proof method is mainly from the idea of Marino and Xu [8, Lemma 2.5]. It is known that the norm of a linear bounded self-adjoint operator $V$ on $H$ is as follows:

$$
\|V\|=\sup \{|\langle V x, x\rangle|: x \in H,\|x\|=1\} .
$$


Now, for all $x \in H$ with $\|x\|=1$, we see that (here 0 denotes zero point in $H$ )

$$
\begin{aligned}
\|I-\rho A\| & =\sup \{\langle(I-\rho A) x, x\rangle: x \in H,\|x\|=1\} \\
& =\sup \{\langle(I-\rho A) x-(I-\rho A) 0, x-0\rangle: x \in H,\|x\|=1\} \\
& =\sup \{\langle(x-0)-\rho(A x-A 0), x-0\rangle: x \in H,\|x\|=1\} \\
& =\sup \left\{\|x\|^{2}-\rho\langle(A x-A 0), x-0\rangle: x \in H,\|x\|=1\right\} \\
& \leq \sup \left\{1-\rho \alpha\|x-0\|^{2}: x \in H,\|x\|=1\right\} \\
& =1-\rho \alpha .
\end{aligned}
$$

This completes the proof.

Remark 2.6. Lemma 2.5 still holds if $A$ is a strongly positive linear bounded operator (see [8, Lemma 2.5]). That is, Lemma 2.5 in this section and Lemma 2.5 in [8] both hold when $A$ is a strongly monotone linear bounded operator or a strongly positive linear bounded one because an operator on a Hilbert space is strongly monotone linear if and only if it is strongly positive linear.

In fact, if $A$ is a strongly monotone linear operator with coefficient $\alpha>0$ on a Hilbert space $H$, then, for all $x \in H$,

$$
\langle A x, x\rangle=\langle A x-A 0, x-0\rangle \geq \alpha\|x-0\|^{2}=\alpha\|x\|^{2},
$$

which shows that $A$ is strongly positive linear. Assume that $A$ is a strongly positive linear operator with coefficient $\alpha>0$ on $H$. Then, for all $x, y \in H$,

$$
\langle A x-A y, x-y\rangle=\langle A(x-y), x-y\rangle \geq \alpha\|x-y\|^{2}
$$

which shows that $A$ is strongly monotone and linear.

\section{Main Results}

Let $H$ be a Hilbert space and $C$ be a nonempty closed and convex subset of $H$. Let $f: H \rightarrow H$ be a contraction with coefficient $0<\alpha<1$. Let $A, B: H \rightarrow H$ be strongly positive linear bounded operator with coefficient $\eta \in(0,1)$ and $\beta>0$, respectively. Take a fixed number $\gamma$ such that $0 \leq \gamma \alpha<\beta$. Then, from Lemma 2.4, it follows that $B-\gamma f$ is strongly monotone with 
coefficient $\beta-\gamma \alpha>0$. For any fixed numbers $\sigma_{1} \in(0,1]$ and $\sigma_{2} \in\left(\left(1-\eta \sigma_{1}\right) /(\beta-\gamma \alpha),(2-\right.$ $\left.\eta \sigma_{1}\right) /(\beta-\gamma \alpha)$ ), we have $\theta=\eta \sigma_{1}-1+\sigma_{2}(\beta-\gamma \alpha) \in(0,1)$, which can be seen easily from the following:

$$
\begin{aligned}
\sigma_{2}<\frac{2-\eta \sigma_{1}}{\beta-\gamma \alpha} & \Longleftrightarrow \sigma_{2}(\beta-\gamma \alpha)<2-\eta \sigma_{1} \\
& \Longleftrightarrow \theta=\eta \sigma_{1}-1+\sigma_{2}(\beta-\gamma \alpha)<1, \\
\frac{1-\eta \sigma_{1}}{\beta-\gamma \alpha}<\sigma_{2} & \Longleftrightarrow \sigma_{2}(\beta-\gamma \alpha)+\eta \sigma_{1}>1 \\
& \Longleftrightarrow \theta=\eta \sigma_{1}-1+\sigma_{2}(\beta-\gamma \alpha)>0 .
\end{aligned}
$$

Moreover, observe that

$$
\begin{aligned}
\left\|\left(\sigma_{1} A-I+\sigma_{2}(B-\gamma f)\right) x-\left(\sigma_{1} A-I+\sigma_{2}(B-\gamma f)\right) y\right\| \\
\quad=\left\|\left(\sigma_{1} A-I\right)(x-y)+\sigma_{2}(B-\gamma f)(x-y)\right\| \\
\leq\left\|\sigma_{1} A-I\right\|\|x-y\|+\sigma_{2}[\|B(x-y)\|+\gamma\|f x-f y\|] \\
\leq\left[\left\|\sigma_{1} A-I\right\|+\sigma_{2}(\|B\|+\gamma \alpha)\right]\|x-y\|,
\end{aligned}
$$

which implies that $\sigma_{1} A-I+\sigma_{2}(B-\gamma f)$ is Lipschitzian with coefficient $\left\|\sigma_{1} A-I\right\|+\sigma_{2}(\|B\|+\gamma \alpha)>$ 0 .

On the other hand, from Lemma 2.4, it follows that

$$
\begin{aligned}
& \left\langle\left(\sigma_{1} A-I+\sigma_{2}(B-\gamma f)\right) x-\left(\sigma_{1} A-I+\sigma_{2}(B-\gamma f)\right) y, x-y\right\rangle \\
& \quad=\sigma_{1}\langle A x-A y, x-y\rangle+\sigma_{2}\langle(B-\gamma f) x-(B-\gamma f) y, x-y\rangle-\|x-y\|^{2} \\
& \quad \geq \sigma_{1} \eta\|x-y\|^{2}+\sigma_{2}(\beta-\gamma \alpha)\|x-y\|^{2}-\|x-y\|^{2} \\
& \quad=\theta\|x-y\|^{2},
\end{aligned}
$$

which implies that $\sigma_{1} A-I+\sigma_{2}(B-\gamma f)$ is strongly monotone with coefficient $\theta>0$. Hence the variational inequality (for short, $\operatorname{VI}\left(\sigma_{1} A-I+\sigma_{2}(B-\gamma f), C\right)$ )

$$
\left\langle\sigma_{1} A-I+\sigma_{2}(B-\gamma f) x^{*}, x-x^{*}\right\rangle \geq 0, \quad \forall x \in C
$$

has the unique solution.

Let $T: H \rightarrow H$ be a nonexpansive mapping. Take two fixed numbers $\mu_{1}$ and $\mu_{2}$ such that $\mu_{1} \in(0,1]$ and $\mu_{2} \in\left(0, \min \left\{1,\|B\|^{-1}\right\}\right]$ and, for all $\lambda \in\left(0, \min \left\{1,\|A\|^{-1} / \mu_{1}\right\}\right)$, define a mapping $T^{\curlywedge}: H \rightarrow H$ by

$$
T^{\lambda} x=\left(I-\lambda \mu_{1} A\right) T x+\lambda\left[T x-\mu_{2}(B T x-\gamma f(x))\right], \quad \forall x \in H .
$$

Then we have the following results. 
Lemma 3.1. If $\mu_{2} \in\left(\left(1-\eta \mu_{1}\right) /(\beta-\gamma \lambda),\left(2-\eta \mu_{1}\right) /(\beta-\gamma \lambda)\right)$, then $T^{\lambda}$ is a contraction with coefficient $1-\lambda \tau$, where $\tau=\eta \mu_{1}-1+\mu_{2}(\beta-\gamma \alpha) \in(0,1)$, that is,

$$
\left\|T^{\curlywedge} x-T^{\lambda} y\right\| \leq(1-\lambda \tau)\|x-y\|, \quad \forall x, y \in H
$$

Proof. From Lemma 2.5 and Remark 2.6, it follows that, for all $x, y \in H$,

$$
\begin{aligned}
\left\|T^{\lambda} x-T^{\lambda} y\right\|= & \|\left(I-\lambda \mu_{1} A\right) T x+\lambda\left[T x-\mu_{2}(B T x-\gamma f(x))\right] \\
& -\left(I-\lambda \mu_{1} A\right) T y-\lambda\left[T y-\mu_{2}(B T y-\gamma f(y))\right] \| \\
\leq & \left\|\left(I-\lambda \mu_{1} A\right) T x-\left(I-\lambda \mu_{1} A\right) T y\right\| \\
& +\lambda\left\|T x-\mu_{2}(B T x-\gamma f(x))-\left[T y-\mu_{2}(B T y-\gamma f(y))\right]\right\| \\
\leq & \left\|\left(I-\lambda \mu_{1} A\right)\right\|\|T x-T y\| \\
& +\lambda\left[\left\|\left(I-\mu_{2} B\right) T x-\left(I-\mu_{2} B\right) T y\right\|+\mu_{2} \gamma\|f(x)-f(y)\|\right] \\
\leq & \left(1-\lambda \mu_{1} \eta\right)\|x-y\|+\lambda\left[\left\|I-\mu_{2} B\right\|\|T x-T y\|+\mu_{2} \gamma \alpha\|x-y\|\right] \\
\leq & \left\{1-\lambda \mu_{1} \eta+\lambda\left[1-\mu_{2}(\beta-\gamma \alpha)\right]\right\}\|x-y\| \\
= & \left\{1-\lambda\left[\mu_{1} \eta-1+\mu_{2}(\beta-\gamma \alpha)\right]\right\}\|x-y\| \\
= & (1-\lambda \tau)\|x-y\| .
\end{aligned}
$$

This completes the proof.

Let $\left\{T_{i}\right\}_{n=1}^{\infty}$ be a countable family of nonexpansive mappings from $H$ into itself such that $C=\bigcap_{n=1}^{\infty} F\left(T_{i}\right) \neq \emptyset$. Since each $F\left(T_{i}\right)$ is closed and convex, then $C$ is closed and convex.

Throughout this paper, let $f: H \rightarrow H$ be a contraction with coefficient $0<\alpha<1$. Let $A, B: H \rightarrow H$ be strongly positive linear bounded mapping with coefficient $\eta \in(0,1)$ and $\beta>0$, respectively. Take a fixed number $\gamma$ such that $0<\gamma \alpha<\beta$. Suppose that $\mu_{1} \in(0,1]$, $\mu_{2} \in\left(\left(1-\eta \mu_{1}\right) /(\beta-\gamma \alpha), \min \left\{1,\|B\|^{-1},\left(2-\eta \mu_{1}\right) /(\beta-\gamma \alpha)\right\}\right)$ (assuming that $\left(1-\eta \mu_{1}\right) /(\beta-\gamma \alpha)<$ $\min \left\{1,\|B\|^{-1}\right\}$ such that $\left(\left(1-\eta \mu_{1}\right) /(\beta-\gamma \lambda), \min \left\{1,\|B\|^{-1},\left(2-\eta \mu_{1}\right) /(\beta-\gamma \lambda)\right\}\right]$ is nonempty), $\left\{\lambda_{n}\right\}_{n=1}^{\infty} \subset\left(0, \min \left\{1,\|A\|^{-1} / \mu_{1}\right\}\right)$ with $\liminf _{n \rightarrow \infty} \lambda_{n}>0$ and $\left\{\alpha_{n}\right\}_{n=0}^{\infty} \subset(0,1]$ with $\alpha_{0}=1$.

Now, we can rewrite the iterative scheme (1.12) as follows:

$$
x_{n+1}=\alpha_{n} T^{\lambda_{n}} x_{n}+\sum_{i=1}^{n}\left(\alpha_{i-1}-\alpha_{i}\right) T_{i} x_{n}, \quad \forall n \geq 1,
$$

where $T^{\lambda_{n}} x_{n}=\left(I-\lambda_{n} \mu_{1} A\right) x_{n}+\lambda_{n}\left[x_{n}-\mu_{2}\left(B x_{n}-\gamma f\left(x_{n}\right)\right)\right]$. Then, by Lemma 3.1, for all $x, y \in H$, we have

$$
\left\|T_{n}^{\lambda_{n}} x-T_{n}^{\lambda_{n}} y\right\| \leq\left(1-\lambda_{n} \tau\right)\|x-y\|, \quad \forall n \geq 1,
$$

where $\tau=\eta \mu_{1}-1+\mu_{2}(\beta-\gamma \alpha) \in(0,1)$. 
Journal of Inequalities and Applications

Lemma 3.2. If $\left\{\alpha_{n}\right\}$ is strictly decreasing, then the scheme $\left\{x_{n}\right\}$ defined by (3.9) is bounded.

Proof. Since $\left\|T^{\lambda_{n}} p-p\right\|=\lambda_{n}\left\|\left(\mu_{1} A-I+\mu_{2}(B-\gamma f)\right) p\right\|$, it follows from (3.10) that, for all $p \in C$,

$$
\begin{aligned}
\left\|x_{n+1}-p\right\| & =\left\|\alpha_{n}\left(T^{\lambda_{n}} x_{n}-p\right)+\sum_{i=1}^{n}\left(\alpha_{i-1}-\alpha_{i}\right)\left(T_{i} x_{n}-p\right)\right\| \\
& \leq \alpha_{n}\left\|T^{\lambda_{n}} x_{n}-p\right\|+\sum_{i=1}^{n}\left(\alpha_{i-1}-\alpha_{i}\right)\left\|T_{i} x_{n}-p\right\| \\
& \leq \alpha_{n}\left\|T^{\lambda_{n}} x_{n}-T^{\lambda_{n}} p\right\|+\alpha_{n}\left\|T^{\lambda_{n}} p-p\right\|+\left(1-\alpha_{n}\right)\left\|x_{n}-p\right\| \\
& \leq \alpha_{n}\left(1-\lambda_{n} \tau\right)\left\|x_{n}-p\right\|+\alpha_{n} \lambda_{n}\left\|\left(\mu_{1} A-I+\mu_{2}(B-\gamma f)\right) p\right\|+\left(1-\alpha_{n}\right)\left\|x_{n}-p\right\| \\
& =\left(1-\alpha_{n} \lambda_{n} \tau\right)\left\|x_{n}-p\right\|+\alpha_{n} \lambda_{n}\left\|\left(\mu_{1} A-I+\mu_{2}(B-\gamma f)\right) p\right\| .
\end{aligned}
$$

By induction, we obtain

$$
\left\|x_{n+1}-p\right\| \leq \max \left\{\left\|x_{1}-p\right\|, \frac{1}{\tau}\left\|\left(\mu_{1} A-I+\mu_{2}(B-\gamma f)\right) p\right\|\right\} .
$$

Hence $\left\{x_{n}\right\}$ is bounded and so are $\left\{T^{\lambda_{n}} x_{n}\right\}$ and $\left\{T_{i} x_{n}\right\}$ for each $i \geq 1$. This completes the proof.

Lemma 3.3. If $\left\{\alpha_{n}\right\}$ is strictly decreasing and the following conditions hold:

$$
\sum_{n=1}^{\infty} \alpha_{n}=\infty, \quad \sum_{n=1}^{\infty}\left|\lambda_{n}-\lambda_{n+1}\right|<\infty,
$$

then $\lim _{n \rightarrow \infty}\left\|x_{n+1}-x_{n}\right\|=0$.

Proof. By the iterative scheme (3.9), we have

$$
\begin{aligned}
x_{n+1}-x_{n}= & \alpha_{n} T^{\lambda_{n}} x_{n}+\sum_{i=1}^{n}\left(\alpha_{i-1}-\alpha_{i}\right) T_{i} x_{n}-\left(\alpha_{n-1} T^{\lambda_{n-1}} x_{n-1}+\sum_{i=1}^{n-1}\left(\alpha_{i-1}-\alpha_{i}\right) T_{i} x_{n-1}\right) \\
= & \alpha_{n}\left(T^{\lambda_{n}} x_{n}-T^{\lambda_{n}} x_{n-1}\right)+\sum_{i=1}^{n}\left(\alpha_{i-1}-\alpha_{i}\right)\left(T_{i} x_{n}-T_{i} x_{n-1}\right) \\
& +\sum_{i=1}^{n}\left(\alpha_{i-1}-\alpha_{i}\right) T_{i} x_{n-1}-\sum_{i=1}^{n-1}\left(\alpha_{i-1}-\alpha_{i}\right) T_{i} x_{n-1}+\alpha_{n} T^{\lambda_{n}} x_{n-1}-\alpha_{n-1} T^{\lambda_{n-1}} x_{n-1} \\
= & \alpha_{n}\left(T^{\lambda_{n}} x_{n}-T^{\lambda_{n}} x_{n-1}\right)+\sum_{i=1}^{n}\left(\alpha_{i-1}-\alpha_{i}\right)\left(T_{i} x_{n}-T_{i} x_{n-1}\right) \\
& +\left(\alpha_{n-1}-\alpha_{n}\right) T_{n} x_{n-1}+\left(\alpha_{n-1} \lambda_{n-1}-\alpha_{n} \lambda_{n}\right)\left[\left(\mu_{1} A-I+\mu_{2}(B-\gamma f)\right) x_{n-1}\right] \\
& +\left(\alpha_{n}-\alpha_{n-1}\right) x_{n-1}
\end{aligned}
$$




$$
\begin{aligned}
= & \alpha_{n}\left(T^{\lambda_{n}} x_{n}-T^{\lambda_{n}} x_{n-1}\right)+\sum_{i=1}^{n}\left(\alpha_{i-1}-\alpha_{i}\right)\left(T_{i} x_{n}-T_{i} x_{n-1}\right) \\
& +\left(\alpha_{n-1}-\alpha_{n}\right) T_{n} x_{n-1}+\left(\alpha_{n}-\alpha_{n-1}\right) x_{n-1} \\
& +\left[\left(\alpha_{n-1}-\alpha_{n}\right) \lambda_{n}+\left(\lambda_{n-1}-\lambda_{n}\right) \alpha_{n-1}\right]\left[\left(\mu_{1} A-I+\mu_{2}(B-\gamma f)\right) x_{n-1}\right]
\end{aligned}
$$

and hence

$$
\begin{aligned}
\left\|x_{n+1}-x_{n}\right\| \leq & \alpha_{n}\left\|T^{\lambda_{n}} x_{n}-T^{\lambda_{n}} x_{n-1}\right\|+\sum_{i=1}^{n}\left(\alpha_{i-1}-\alpha_{n}\right)\left\|T_{i} x_{n}-T_{i} x_{n-1}\right\| \\
& +\left(\alpha_{n-1}-\alpha_{n}\right)\left\|T_{n} x_{n-1}\right\|+\left(\alpha_{n-1}-\alpha_{n}\right)\left\|x_{n-1}\right\| \\
& +\left[\left(\alpha_{n-1}-\alpha_{n}\right) \lambda_{n}+\left|\lambda_{n-1}-\lambda_{n}\right| \alpha_{n-1}\right]\left\|\left(\mu_{1} A-I+\mu_{2}(B-\gamma f)\right) x_{n-1}\right\| \\
\leq & \alpha_{n}\left(1-\lambda_{n} \tau\right)\left\|x_{n}-x_{n-1}\right\|+\left(1-\alpha_{n}\right)\left\|x_{n}-x_{n-1}\right\| \\
& +\left(\alpha_{n-1}-\alpha_{n}\right)\left\|T_{n} x_{n-1}\right\|+\left(\alpha_{n-1}-\alpha_{n}\right)\left\|x_{n-1}\right\| \\
& +\left[\left(\alpha_{n-1}-\alpha_{n}\right)+\left|\lambda_{n-1}-\lambda_{n}\right|\right]\left\|\left(\mu_{1} A-I+\mu_{2}(B-\gamma f)\right) x_{n-1}\right\| \\
\leq & \left(1-\alpha_{n} \lambda_{n} \tau\right)\left\|x_{n}-x_{n-1}\right\|+\left(\alpha_{n-1}-\alpha_{n}\right) M+\left|\lambda_{n-1}-\lambda_{n}\right| M,
\end{aligned}
$$

where $M$ is a constant. Since $\left\{\lambda_{n}\right\} \subset\left(0, \min \left\{1,\|A\|^{-1} / \mu_{1}\right\}\right)$, there exists a constant $\lambda^{\prime}>0$ such that $\lambda_{n} \geq \lambda^{\prime}$ for all $n \geq 1$. Therefore, we have

$$
\left\|x_{n+1}-x_{n}\right\| \leq\left(1-\alpha_{n} \lambda^{\prime} \tau\right)\left\|x_{n}-x_{n-1}\right\|+\left[\left(\alpha_{n-1}-\alpha_{n}\right)+\left|\lambda_{n-1}-\lambda_{n}\right|\right] M
$$

Put $c_{n}=\left[\left(\alpha_{n-1}-\alpha_{n}\right)+\left|\lambda_{n-1}-\lambda_{n}\right|\right] M$. Since $\left\{\alpha_{n}\right\}$ is a strictly decreasing sequence and $\sum_{n=2}^{\infty} \mid \lambda_{n-1}-$ $\lambda_{n} \mid<\infty$, we have $\sum_{n=2}^{\infty} c_{n}<\infty$. By Lemma 2.3, it follows that $\left\|x_{n+1}-x_{n}\right\| \rightarrow 0$ as $n \rightarrow \infty$. This completes the proof.

Lemma 3.4. If $\left\{\alpha_{n}\right\}$ is strictly decreasing and the following conditions hold:

$$
\lim _{n \rightarrow \infty} \alpha_{n}=0, \quad \sum_{n=1}^{\infty} \alpha_{n}=\infty, \quad \sum_{n=1}^{\infty}\left|\lambda_{n}-\lambda_{n+1}\right|<\infty
$$

then $\lim _{n \rightarrow \infty}\left\|x_{n}-T_{i} x_{n}\right\|=0$, for all $i \geq 1$.

Proof. By the iterative scheme (3.9), we have

$$
x_{n+1}+\sum_{i=1}^{n}\left(\alpha_{i-1}-\alpha_{i}\right)\left(x_{n}-T_{i} x_{n}\right)-\left(1-\alpha_{n}\right) x_{n}=\alpha_{n} T^{\lambda_{n}} x_{n}
$$


that is,

$$
\sum_{i=1}^{n}\left(\alpha_{i-1}-\alpha_{i}\right)\left(x_{n}-T_{i} x_{n}\right)=\alpha_{n}\left(T^{\lambda_{n}} x_{n}-x_{n}\right)+\left(x_{n}-x_{n+1}\right) .
$$

Hence, for any $p \in C$, we get

$$
\sum_{i=1}^{n}\left(\alpha_{i-1}-\alpha_{i}\right)\left\langle x_{n}-T_{i} x_{n}, x_{n}-p\right\rangle=\alpha_{n}\left\langle T^{\lambda_{n}} x_{n}-x_{n}, x_{n}-p\right\rangle+\left\langle x_{n}-x_{n+1}, x_{n}-p\right\rangle .
$$

Since each $T_{i}$ is nonexpansive, it follows from (2.2) that

$$
\left\|T_{i} x_{n}-x_{n}\right\|^{2} \leq 2\left\langle x_{n}-T_{i} x_{n}, x_{n}-p\right\rangle .
$$

Hence, combining (3.21) with (3.20), it follows that

$$
\begin{aligned}
\sum_{i=1}^{n}\left(\alpha_{i-1}-\alpha_{i}\right)\left\|T_{i} x_{n}-x_{n}\right\|^{2} & \leq 2 \sum_{i=1}^{n}\left(\alpha_{i-1}-\alpha_{i}\right)\left\langle x_{n}-T_{i} x_{n}, x_{n}-p\right\rangle \\
& =2 \alpha_{n}\left\langle T^{\lambda_{n}} x_{n}-x_{n}, x_{n}-p\right\rangle+2\left\langle x_{n}-\mathrm{x}_{n+1}, x_{n}-p\right\rangle,
\end{aligned}
$$

which implies that

$$
\frac{1}{2} \sum_{i=1}^{n}\left(\alpha_{i-1}-\alpha_{i}\right)\left\|T_{i} x_{n}-x_{n}\right\|^{2} \leq \alpha_{n}\left\langle T^{\lambda_{n}} x_{n}-x_{n}, x_{n}-p\right\rangle+\left\langle x_{n}-x_{n+1}, x_{n}-p\right\rangle .
$$

Since each $\left(\alpha_{i-1}-\alpha_{i}\right)\left\|T_{i} x_{n}-x_{n}\right\|^{2} \geq 0$ and $\alpha_{i-1}-\alpha_{i}>0$, then we have

$$
\frac{1}{2}\left(\alpha_{i-1}-\alpha_{i}\right)\left\|T_{i} x_{n}-x_{n}\right\|^{2} \leq \alpha_{n}\left\langle T^{\lambda_{n}} x_{n}-x_{n}, x_{n}-p\right\rangle+\left\langle x_{n}-x_{n+1}, x_{n}-p\right\rangle,
$$

that is,

$$
\left\|T_{i} x_{n}-x_{n}\right\|^{2} \leq \frac{2 \alpha_{n}}{\alpha_{i-1}-\alpha_{i}}\left\langle T^{\lambda_{n}} x_{n}-x_{n}, x_{n}-p\right\rangle+\frac{2}{\alpha_{i-1}-\alpha_{i}}\left\langle x_{n}-x_{n+1}, x_{n}-p\right\rangle .
$$

Since $\left\{x_{n}\right\}$ and $\left\{T^{\lambda_{n}} x_{n}\right\}$ are both bounded, there exists a constant $M^{\prime}>0$ such that

$$
\left\|T_{i} x_{n}-x_{n}\right\|^{2} \leq M^{\prime}\left(\frac{\alpha_{n}}{\alpha_{i-1}-\alpha_{i}}+\frac{1}{\alpha_{i-1}-\alpha_{i}}\left\|x_{n}-x_{n+1}\right\|\right) .
$$

By Lemma 3.3 and the assumption condition $\lim _{n \rightarrow \infty} \alpha_{n}=0$, it follows that

$$
\lim _{n \rightarrow \infty}\left\|T_{i} x_{n}-x_{n}\right\|=0, \quad \forall i \geq 1 .
$$

This completes the proof. 
Finally, we give the main result in this paper.

Theorem 3.5. If $\left\{\alpha_{n}\right\}$ is strictly decreasing and the following conditions hold:

$$
\lim _{n \rightarrow \infty} \alpha_{n}=0, \quad \sum_{n=1}^{\infty} \alpha_{n}=\infty, \quad \sum_{n=1}^{\infty}\left|\lambda_{n}-\lambda_{n+1}\right|<\infty,
$$

then the scheme $\left\{x_{n}\right\}$ defined by (3.9) converges strongly to an element $x^{*} \in C$ which is the unique solution of the variational inequality $\left(\operatorname{VI}\left(\mu_{1} A-I+\mu_{2}(B-\gamma f), C\right)\right)$ :

$$
\left\langle\left[\mu_{1} A-I+\mu_{2}(B-\gamma f)\right] x^{*}, x-x^{*}\right\rangle \geq 0, \quad \forall x \in C .
$$

Proof. First, we prove that $\limsup _{n \rightarrow \infty}\left\langle-\left[\mu_{1} A-I+\mu_{2}(B-\gamma f)\right] x^{*}, x_{n+1}-x^{*}\right\rangle \leq 0$.

To prove this, we pick a subsequence $\left\{x_{n_{i}}\right\}$ of $\left\{x_{n}\right\}$ such that

$$
\limsup _{n \rightarrow \infty}\left\langle-\left[\mu_{1} A-I+\mu_{2}(B-\gamma f)\right] x^{*}, x_{n}-x^{*}\right\rangle=\lim _{i \rightarrow \infty}\left\langle-\left[\mu_{1} A-I+\mu_{2}(B-\gamma f)\right] x^{*}, x_{n_{i}}-x^{*}\right\rangle .
$$

Without loss of generality, we may, further, assume that $x_{n_{i}} \rightarrow \widehat{x}$ for some $\widehat{x} \in H$. From Lemmas 2.1 and 3.3, it follows that $\widehat{x} \in F\left(T_{i}\right)$ for each $i \geq 1$ and so $\widehat{x} \in C=\cap_{i=1}^{\infty} F\left(T_{i}\right)$. Since $x^{*}$ is the unique solution of the problem $\operatorname{VI}\left(\mu_{1} A-I+\mu_{2}(B-\gamma f), C\right)$, we obtain

$$
\limsup _{n \rightarrow \infty}\left\langle-\left[\mu_{1} A-I+\mu_{2}(B-\gamma f)\right] x^{*}, x_{n}-x^{*}\right\rangle=\left\langle-\left[\mu_{1} A-I+\mu_{2}(B-\gamma f)\right] x^{*}, \widehat{x}-x^{*}\right\rangle \leq 0
$$

It follows from Lemma 2.2 and (3.10) that

$$
\begin{aligned}
\left\|x_{n+1}-x^{*}\right\|^{2}= & \left\|\left[\alpha_{n}\left(T^{\lambda_{n}} x_{n}-T^{\lambda_{n}} x^{*}\right)+\sum_{i=1}^{n}\left(\alpha_{i-1}-\alpha_{n}\right)\left(T_{i} x_{n}-x^{*}\right)\right]+\alpha_{n}\left(T^{\lambda_{n}} x^{*}-x^{*}\right)\right\|^{2} \\
\leq & \left\|\alpha_{n}\left(T^{\lambda_{n}} x_{n}-T^{\lambda_{n}} x^{*}\right)+\sum_{i=1}^{n}\left(\alpha_{i-1}-\alpha_{n}\right)\left(T_{i} x_{n}-x^{*}\right)\right\|^{2} \\
& +2 \alpha_{n}\left\langle T^{\lambda_{n}} x^{*}-x^{*}, x_{n+1}-x^{*}\right\rangle \\
\leq & {\left[\alpha_{n}\left(1-\lambda_{n} \tau\right)\left\|x_{n}-x^{*}\right\|+\left(1-\alpha_{n}\right)\left\|x_{n}-x^{*}\right\|\right]^{2} } \\
& +2 \alpha_{n}\left\langle-\left[\mu_{1} A-I+\mu_{2}(B-\gamma f)\right] x^{*}, x_{n+1}-x^{*}\right\rangle \\
\leq & \left(1-\alpha_{n} \lambda_{n} \tau\right)\left\|x_{n}-x^{*}\right\|^{2}+2 \alpha_{n}\left\langle-\left[\mu_{1} A-I+\mu_{2}(B-\gamma f)\right] x^{*}, x_{n+1}-x^{*}\right\rangle \\
\leq & \left(1-\alpha_{n} \lambda^{\prime} \tau\right)\left\|x_{n}-x^{*}\right\|^{2}+2 \alpha_{n}\left\langle-\left[\mu_{1} A-I+\mu_{2}(B-\gamma f)\right] x^{*}, x_{n+1}-x^{*}\right\rangle,
\end{aligned}
$$


where $\lambda^{\prime}>0$ is a constant such that $\lambda_{n} \geq \lambda^{\prime}$ for all $n \geq 1$. Since $\sum_{n=0}^{\infty} \alpha_{n}=\infty$ and $\limsup _{n \rightarrow \infty}\left\langle-\left[\mu_{1} A-I+\mu_{2}(B-\gamma f)\right] x^{*}, x_{n+1}-x^{*}\right\rangle \leq 0$, by Lemma 2.3, we conclude that the scheme $\left\{x_{n}\right\}$ converges strongly to $x^{*}$. This completes the proof.

Remark 3.6. (1) For each $n \geq 1$, a simple example on control parameters is $\alpha_{n}=1 / n$ and $\lambda_{n}=\lambda$, where $\lambda$ is a constant in $\left(0, \min \left\{1,\|A\|^{-1} / \mu_{1}\right\}\right)$.

(2) We obtain the desired results without any assumptions on the family $\left\{T_{i}\right\}_{i=1}^{\infty}$. Foe example, in Theorem CGY2, the authors gave the strong condition (1.10).

Remark 3.7. (1) If $T_{1}=T_{2}=\cdots=T_{n}=\cdots=T$ in (3.9), then we have the following iterative scheme:

$$
x_{n+1}=\alpha_{n}\left[\left(I-\lambda_{n} \mu_{1} A\right) x_{n}+\lambda_{n}\left(x_{n}-\mu_{2}\left(B x_{n}-\gamma f\left(x_{n}\right)\right)\right)\right]+\left(1-\alpha_{n}\right) T x_{n}, \quad \forall n \geq 1,
$$

and the scheme $\left\{x_{n}\right\}$ defined by (3.33) converges strongly to an element $x^{*} \in C$ which is the unique solution of the variational inequality $\left(\operatorname{VI}\left(\mu_{1} A-I+\mu_{2}(B-\gamma f), C\right)\right)$ :

$$
\left\langle\left[\mu_{1} A-I+\mu_{2}(B-\gamma f)\right] x^{*}, x-x^{*}\right\rangle \geq 0, \quad \forall x \in C .
$$

(2) If $A=I$ and $\mu_{1}=1$ in (3.33), then we have the following iterative scheme:

$$
x_{n+1}=\alpha_{n}\left(I-\lambda_{n} \mu_{2}(B-\gamma f)\right) x_{n}+\sum_{i=1}^{n}\left(\alpha_{i-1}-\alpha_{i}\right) T_{i} x_{n}, \quad \forall n \geq 1,
$$

and the scheme $\left\{x_{n}\right\}$ defined by (3.35) converges strongly to an element $x^{*} \in C$ which is the unique solution of the variational inequality $(\mathrm{VI}(B-\gamma f, C))$ :

$$
\left\langle\left(\mu_{2}(B-\gamma f)\right) x^{*}, x-x^{*}\right\rangle \geq 0, \quad \forall x \in C \text {. }
$$

(3) Furthermore, if $\mu_{2}=1$ and $\gamma=0$ in (3.35), then we have the following iterative scheme:

$$
x_{n+1}=\alpha_{n}\left(I-\lambda_{n} B\right) x_{n}+\sum_{i=1}^{n}\left(\alpha_{i-1}-\alpha_{i}\right) T_{i} x_{n}, \quad \forall n \geq 1,
$$

and the scheme $\left\{x_{n}\right\}$ defined by (3.37) converges strongly to an element $x^{*} \in C$ which is the unique solution of the variational inequality $(\mathrm{VI}(B, C))$, which is Stampacchia's variational inequality:

$$
\left\langle B x^{*}, x-x^{*}\right\rangle \geq 0, \quad \forall x \in C .
$$

\section{Acknowledgment}

This work was supported by the Korea Research Foundation Grant funded by the Korean Government (KRF-2008-313-C00050). 


\section{References}

[1] D. Kinderlehrer and G. Stampacchia, An Introduction to Variational Inequalities and Their Applications, vol. 88 of Pure and Applied Mathematics, Academic Press, New York, NY, USA, 1980.

[2] H.-K. Xu, "Viscosity approximation methods for nonexpansive mappings," Journal of Mathematical Analysis and Applications, vol. 298, no. 1, pp. 279-291, 2004.

[3] F. Deutsch and I. Yamada, "Minimizing certain convex functions over the intersection of the fixed point sets of nonexpansive mappings," Numerical Functional Analysis and Optimization, vol. 19, no. 1-2, pp. 33-56, 1998.

[4] H.-K. Xu, "Iterative algorithms for nonlinear operators," Journal of the London Mathematical Society, vol. 66, no. 1, pp. 240-256, 2002.

[5] H. K. Xu, "An iterative approach to quadratic optimization," Journal of Optimization Theory and Applications, vol. 116, no. 3, pp. 659-678, 2003.

[6] I. Yamada, "The hybrid steepest descent method for the variational inequality problem over the intersection of fixed point sets of nonexpansive mappings," in Inherently Parallel Algorithms in Feasibility and Optimization and Their Applications, D. Butnarium, Y. Censor, and S. Reich, Eds., vol. 8 of Stud. Comput. Math., pp. 473-504, North-Holland, Amsterdam, The Netherlands, 2001.

[7] I. Yamada, N. Ogura, Y. Yamashita, and K. Sakaniwa, "Quadratic optimization of fixed points of nonexpansive mappings in Hilbert space," Numerical Functional Analysis and Optimization, vol. 19, no. 1-2, pp. 165-190, 1998.

[8] G. Marino and H.-K. Xu, "A general iterative method for nonexpansive mappings in Hilbert spaces," Journal of Mathematical Analysis and Applications, vol. 318, no. 1, pp. 43-52, 2006.

[9] L.-C. Ceng, S.-M. Guu, and J.-C. Yao, "Hybrid viscosity-like approximation methods for nonexpansive mappings in Hilbert spaces," Computers E Mathematics with Applications, vol. 58, no. 3, pp. 605-617, 2009.

[10] K. Goebel and W. A. Kirk, Topics in Metric Fixed Point Theory, vol. 28 of Cambridge Studies in Advanced Mathematics, Cambridge University Press, Cambridge, UK, 1990.

[11] P.-E. Maingé, "Approximation methods for common fixed points of nonexpansive mappings in Hilbert spaces," Journal of Mathematical Analysis and Applications, vol. 325, no. 1, pp. 469-479, 2007. 